\title{
Morphological demonstration of the failure of Mus caroli trophoblast in the Mus musculus uterus*
}

\author{
M. A. Crepeau, S. Yamashiro and B. A. Croy \\ Department of Biomedical Sciences, University of Guelph, Guelph, Ontario, Canada NIG $2 W 1$
}

\begin{abstract}
Summary. A histological study of Mus caroli embryos gestating in the Mus musculus uterus was undertaken at Day 8.5 of gestation, 1 day after such embryos are reported to be normal and 1 day before the earliest events associated with death of the xenogeneic embryos. In comparison to control $M$. caroli embryos recovered from $M$. caroli and to control $M$. musculus embryos recovered from $M$. musculus, the xenogeneically transferred embryos showed intrauterine growth retardation that was associated with trophoblastic insufficiency. Trophoblast cell degeneration was observed, in the absence of lymphocytic infiltration. Therefore, loss of trophoblast cell function rather than lymphocyte-mediated destruction of trophoblast appears to underlie the death of $M$. caroli embryos in the $M$. musculus uterus.
\end{abstract}

Keywords: trophoblast; mouse; pregnancy failure/abortion; xenogeneic embryo transfer; embryonic chimaeras

\section{Introduction}

The biological interactions between mother and fetus that ensure successful mammalian pregnancy are numerous and complex. Trophoblast plays a crucial, but incompletely defined, role in the maintenance of pregnancy (Rossant et al., 1983; Surani et al., 1987). Early in its development, trophoblast surrounds the embryo and is actively involved in nutrient provision (Snell \& Stevens, 1966). Later in gestation, trophoblast anchors the conceptus to the uterine wall and forms the bulk of the definitive placenta. Trophoblast has been shown to secrete hormones and growth factors (Sherman, 1983; Linzer \& Nathans, 1985; Adamson, 1987) and is considered to be an immunological barrier between the mother and her immunogenic conceptus (Chaouat et al., 1983; Hunziker \& Wegmann, 1987). Whether any single function of trophoblast controls the success of pregnancy is unclear.

That trophoblast alone can control the success or failure of pregnancy has been demonstrated by successful gestation of chimaeras, composed of xenogeneic embryonic cells protected by trophoblast, species-homologous to the embryo transfer recipient (Rossant et al., 1982, 1983; Fehilly \& Willadsen, 1986; Polzin et al., 1987; Anderson, 1988). In the murine model of interspecies pregnancy, involving $M u$ s caroli and $M u s$ musculus, fully xenogeneic $M$. caroli are viable in $M$. musculus following transfer of chimaeric blastocysts composed of a $M$. caroli inner cell mass within a $M$. musculus trophoblast vesicle (Rossant et al., 1983). Non-manipulated $M$. caroli embryos gestating in the $M$. musculus uterus fail to recruit intrauterine suppressor cells (Clark et al., 1983), become infiltrated by cytotoxic lymphocytes (Croy et al., 1982), then die and resorb during the second half of pregnancy (Croy et al., 1982, 1985). While such studies suggest that immunoprotection could be a major role played by trophoblast, transfer of $M$. caroli embryos to immunocompromised and immunodeficient animals failed to prolong gestation. $M$. caroli embryos die at mid-gestation in transfer recipients treated with the immunosuppressive drug cyclosporin $\mathrm{A}$, in

*Reprint requests to Dr B. A. Croy. 

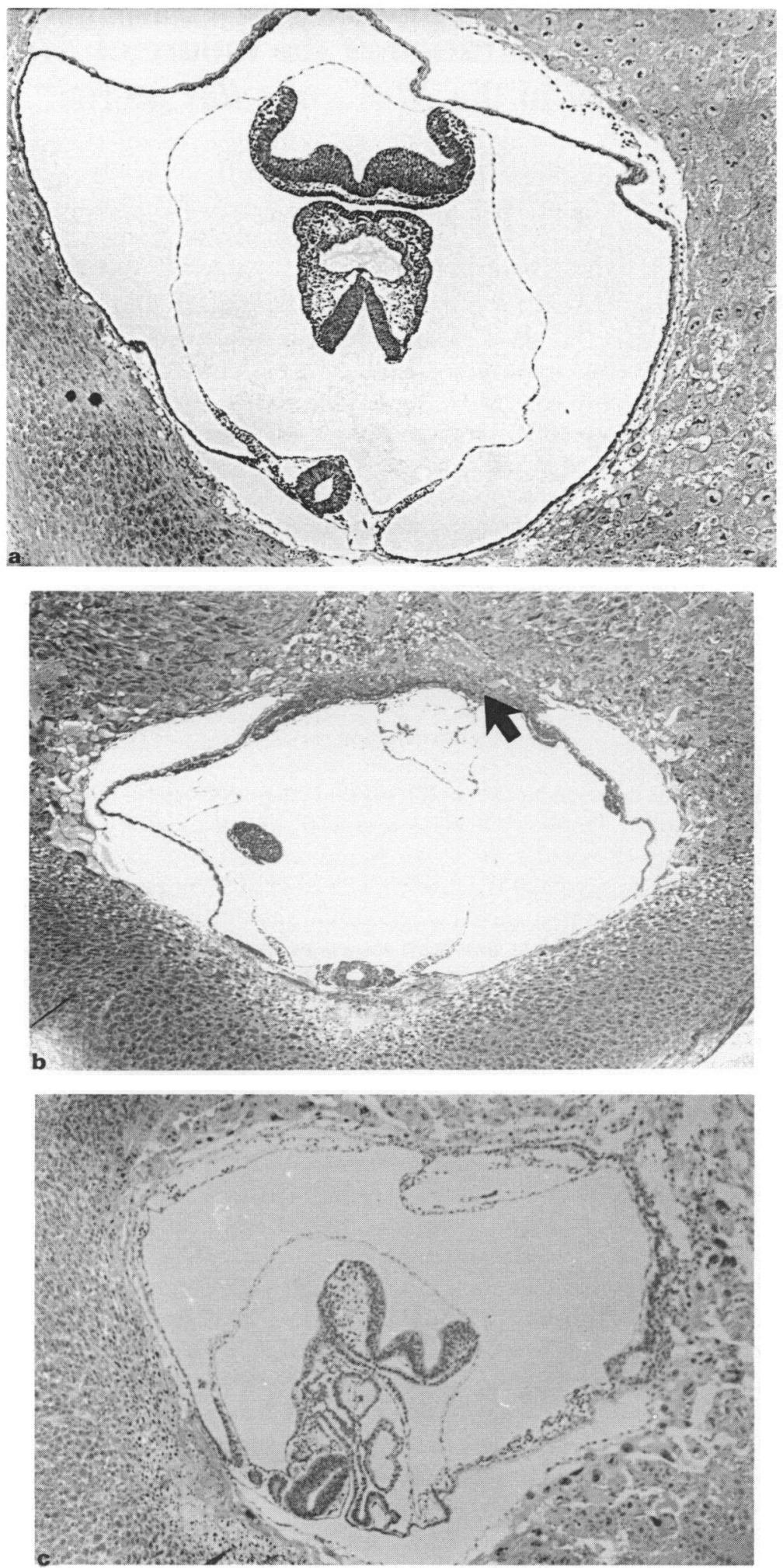
T-lymphocyte-deficient mice of $n u / n u$ genotype, in natural killer cell-deficient mice of $b g / b g$ genotype and in B- and T-cell deficient mice of scid/scid genotype that were unresponsive to antigenic stimulation by $M$. caroli cells in vivo and in vitro (Croy et al., 1985; Crepeau \& Croy, 1988). One interpretation of these observations is that involvement of the immune system in the death and resorption of $M$. caroli in the $M$. musculus uterus is secondary to other, non-immunological problems.

Development of $M$. caroli embryos transferred to $M$. musculus recipients is reported to be normal until Day 7 of gestation (Frels et al., 1980). In a preliminary study assessing chorioallantoic fusion in $M$. caroli embryos transferred to $M$. musculus, abnormalities of trophoblast were detected at Day 8.5 of gestation (Crepeau et al., 1988). An expanded study has therefore been undertaken to characterize $M$. caroli development in the $M$. musculus uterus at Day 8.5 of gestation and to compare it with normal development of $M$. caroli in the $M$. caroli uterus and $M$. musculus in the $M$. musculus uterus.

\section{Materials and Methods}

Mice. Random-bred M. musculus (CD1) were purchased from Charles River Laboratories, St Constant, Quebec, Canada. Random-bred $M$. caroli were bred and maintained at the University of Guelph, Guelph, Ontario, Canada. The $M$. caroli breeding colony was established from stocks obtained in 1978 from Dr V. Chapman, Roswell Park Memorial Institute, Buffalo, NY, USA. Mice were housed under standard conditions and used in experiments at 8-12 weeks of age.

Matings. To obtain $M$. musculus embryos, $M$. musculus females in oestrus were paired overnight with $M$. musculus males. Detection of a vaginal plug the following morning was deemed evidence of successful mating. Pseudopregnant $M$. musculus females were obtained by overnight pairing of oestrous females with vasectomized males, followed by selection of females with vaginal plugs. The morning of plug detection was designated Day 0.5 of pregnancy.

To obtain $M$. caroli embryos, $M$. caroli females were paired with $M$. caroli males after hormone-induced ovulation as previously described (Croy et al., 1982). Since these matings do not produce copulation plugs, all hormone-treated $M$. caroli females were used for embryo collection. Day 0.5 of gestation was $24 \mathrm{~h}$ after administration of human chorionic gonadotrophin (hCG).

Embryo collection and transfer. Blastocysts were collected from M. musculus females at Day 3.5 of gestation, and from $M$. caroli females $76 \mathrm{~h}$ after hCG treatment. Mice were killed by cervical dislocation and the uteri excised and flushed with medium. Blastocysts were transferred to anaesthetized $M$. musculus females at Day 2.5 of pseudopregnancy as previously described (Croy et al., 1982, 1985). Depending upon the number of embryos recovered from the uterine flushings, 3-6 M. caroli blastocysts were transferred into the lumen of one uterine horn and 3-6 $M$. musculus blastocysts to the opposite horn.

Histological analysis of implantation sites. Transferred $M$. caroli and $M$. musculus embryos were recovered from $M$. musculus uteri at Day 8.5 of gestation. Stage-matched untransferred $M$. caroli embryos were recovered from $M$. caroli on Day 7.5 of gestation (Frels et al., 1980).

For light microscopy, implantation sites were individually fixed in 10\% phosphate-buffered formalin for $72 \mathrm{~h}$, and then processed and embedded in glycol methacrylate (Bennett et al., 1976). Serial transverse sections of the implantation sites, 2-3 $\mu \mathrm{m}$ thick, were cut on a JB-4 microtome (Sorvall, Newtown, CN, USA) using glass knives, and then stained with Harris' haematoxylin and eosin or reacted with the periodic acid-Schiff technique (Fisher Scientific, Unionville, Ontario, Canada).

For electron microscopy, small pieces of implantation sites were immersed in a modified Karnovsky's fixative (Karnovsky, 1965) consisting of $4 \%$ paraformaldehyde $(w / v)$ and $2 \%$ glutaraldehyde $(w / v)$ in phosphate-buffered saline $\mathrm{pH} 7.4$ (PBS) for $6 \mathrm{~h}$. The samples were then washed in PBS and post-fixed in $1 \%(\mathrm{w} / \mathrm{v})$ osmium tetroxide (Sigma, St Louis, MO, USA) in PBS, for $2 \mathrm{~h}$. Samples were dehydrated in ethanol and embedded in LX-112 resin (Ladd Research Industries, Burlington, VT, USA). Sections $(1 \mu \mathrm{m})$ were cut with an OMU3 ultramicrotome (Reichert, Vienna, Austria), and areas from these specimens were selected and sectioned for electron microscopy.

Fig. 1. Photomicrographs of transverse sections of implantation sites illustrating development of $M$. caroli embryos; $\times 51$. (a) In $M$. caroli uterus at Day 7.5 , mid-somite stage; (b) in $M$. musculus uterus at Day 8.5 , early somite stage with chorio-allantoic fusion (arrow); and (c) in $M$. musculus uterus at Day $8 \cdot 5$, mid-somite stage with rotation. 

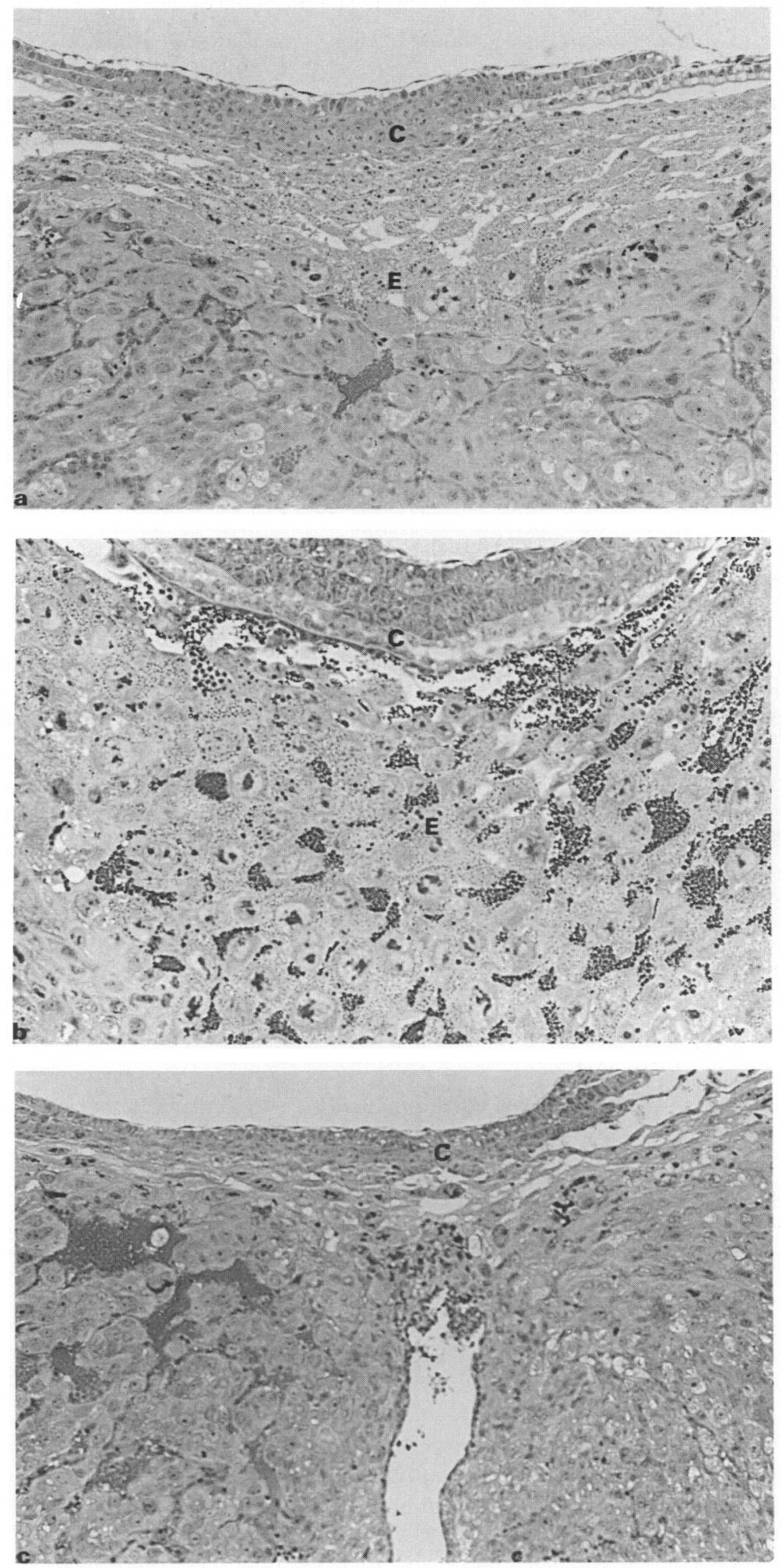
Ultrathin sections were stained with uranyl acetate and lead citrate and examined in a Jeol JEM 100S transmission electron microscope.

\section{Results}

Stage of embryo development in allogeneic and xenogeneic uteri

Five $M$. caroli embryos and $4 M$. musculus embryos recovered from 3 different $M$. musculus recipients, as well as 4 control $M$. caroli embryos recovered from $M$. caroli, were studied using serial sections. An additional $2 M$. caroli embryos and $2 M$. musculus embryos recovered from a single $M$. musculus recipient were processed for transmission electron microscopy.

All of the transferred $M$. musculus embryos had developed normally to the early or mid-somite stage characterized by fusion of the allantois to the chorion and rotation of the embryo (Theiler, 1972). Development of control $M$. caroli embryos gestating in $M$. caroli appeared uniform and normal: 3 were at the mid-somite stage and had rotated while development of the 4th was only slightly retarded (Fig. 1a). In contrast, the stage of development of transferred $M$. caroli embryos was not uniform: 1 embryo was presomite stage with an emerging allantois while another $M$. caroli embryo, from the same uterus, was very early somite stage. In this embryo, the allantois was clearly fused with the chorion (Fig. 1b). The other $M$. caroli embryos, recovered from 2 different $M$. musculus recipients, had developed further and 2 had reached the mid-somite stage with rotation (Fig. 1c).

\section{Trophoblast development in allogeneic and xenogeneic uteri}

Trophoblast development in $M$. musculus embryos was extensive; ectoplacental cones were covered by several layers of polar trophoblast giant cells (Fig. 2a). Mural trophoblast giant cells formed an uninterrupted meshwork between Reichert's membrane and the maternal decidua. In control $M$. caroli embryos, trophoblast was somewhat less abundant than in $M$. musculus but mural giant cells, polar giant cells and ectoplacental cone trophoblast cells could be distinguished (Fig. 2b). By comparison, trophoblast development of transferred $M$. caroli embryos recovered from $M$. musculus uteri was deficient. A few irregular layers of polar trophoblast giant cells were observed above the ectoplacental cones. Numbers of trophoblast giant cells were reduced. In one implantation site, the paucity of trophoblast was drastic and the characteristic ectoplacental cone, seen in normal pregnancies, consisted of only a few islands of cells (Fig. 2c).

\section{Morphological features of trophoblast}

M. musculus ectoplacental cone cells and trophoblast giant cells were densely packed with intensely staining, eosinophilic granules of variable size (Fig. 3a). Similar granules were also abundant in ectoplacental cone cells of control $M$. caroli embryos (Fig. 3b) but were less numerous in the mural and polar trophoblast giant cells. These granules were also stained, although less intensely, using the periodic acid-Schiff's technique. In contrast, granules were markedly deficient in ectoplacental cone cells and trophoblast giant cells from all $M$. caroli embryos recovered from a M. musculus uterus (Fig. 3c).

Transmission electron microscopy was used to characterize further the nature of these cytoplasmic granules. The size and appearance of the granules in M. musculus trophoblast varied;

Fig. 2. Photomicrographs of ectoplacental cone $(E)$ in region of the chorionic plate $(C) ; \times 128$. (a) M. musculus in M. musculus at Day 8.5 with well developed ectoplacental cone; (b) $M$. caroli in $M$. caroli at Day $7 \cdot 5$; and (c) $M$. caroli in $M$. musculus at Day $8 \cdot 5$ showing few trophoblast cells. 

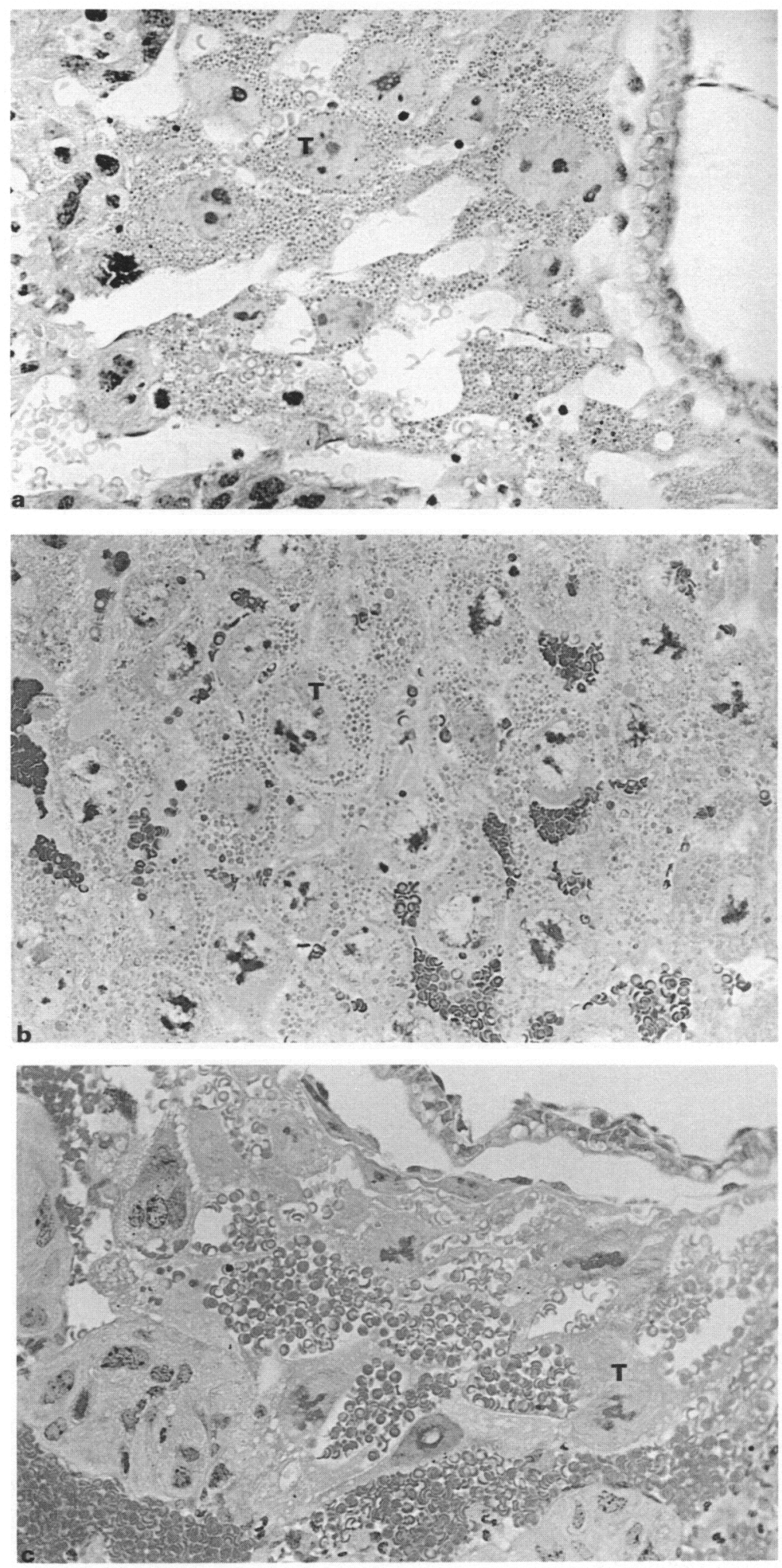
homogeneous electron-dense granules of variable size were most abundant (Fig. 4a). Granules of various electron densities and variable sizes which contained vacuolations were less numerous. Granules of uniform size, composed of fine particulate material of medium electron density, and granules of similar size and appearance, but also containing fine electron-opaque regions, were observed. These appeared to be phagocytosed erythrocytes, not yet fused with lysosomes, and partly digested erythrocytes respectively. Granules present in $M$. caroli trophoblast giant cells were different. Most of those present were large, irregularly shaped and of light to medium electron density, resembling lipid inclusions (Fig. 4b). Intact phagocytosed erythrocytes were also observed. The electron-dense inclusions, abundant in $M$. musculus trophoblast giant cells, were infrequent in trophoblast cells from transferred $M$. caroli embryos.

A second difference was observed between trophoblast giant cells from control and experimental embryos at the light microscopic level. In each of the $M$. caroli embryos recovered from $M$. musculus uteri many giant cells appeared to be in a degenerative state, characterized by ill-defined cellular outlines, vacuolated cytoplasm, pycnosis or karyorrhexis. Mildly eosinophilic exudates were found in areas that appeared devoid of trophoblast giant cells and were next to giant cells that appeared to be dying (Fig. 5). The exudative foci were infiltrated by polymorphs (Fig. 5b) and abutted the yolk sac, particularly in the posterior neural tube region. In the most severely growthretarded $M$. caroli embryo studied, an engorged blood vessel appeared to disrupt the giant cell barrier. In this implantation site, lymphocytes were detected within blood spaces but not within embryonic tissues. In control embryos, neither giant cells with a degenerative appearance nor large exudative foci were observed.

\section{Discussion}

Studies of chimaeric mammalian embryos derived from different species have shown that trophoblast genotype is critical for embryonic success (Rossant et al., 1982, 1983; Fehilly \& Willadsen, 1986; Polzin et al., 1987). While trophoblast has been identified as the protective tissue, the mechanism by which protection is afforded is unknown. New observations obtained from this histological study support the hypothesis that in the $M$. caroli-M. musculus model system, degenerative events affecting $M$. caroli trophoblast precede involvement of immune effector cells in the failure and resorption of $M$. caroli embryos in the xenogeneic uterus.

At Day 8.5 of gestation, retarded, asynchronous development of $M$. caroli embryos in $M$. musculus was deteted in comparison to the stage of development of $M$. caroli or $M$. musculus embryos from intraspecies pregnancies. The inner-cell mass-derived structures of the embryo proper appeared normal in all xenogeneic pregnancies. From earlier studies it is known that these tissues become disorganized then necrotic and collapse before haemorrhagic resorption between Days 10 and 15 (Croy et al., 1982). Such a sequence of events suggests that the lesions present at Day 8.5 of gestation lead progressively to failure of the entire conceptus and excludes mechanisms of generalized embryo toxicity. Variations in the stages of early $M$. caroli development no doubt contribute to the asynchrony of grossly detectable embryonic death within and between $M$. musculus recipients and to the relatively long time interval over which resorptions appear to be initiated (Croy et al., 1985).

Fig. 3. Differences in the appearance of granules within trophoblast cells $(\mathrm{T}) ; \times 320$. (a) $M$. musculus in $M$. musculus at Day 8.5 , with abundant granules of variable size in cytoplasm of trophoblast cells; (b) $M$. caroli, in $M$. caroli at Day 7.5, with granules similar to those of $M$. musculus; and (c) $M$. caroli in $M$. musculus at Day $8 \cdot 5$, showing a relative absence of granules compared to control embryos. 

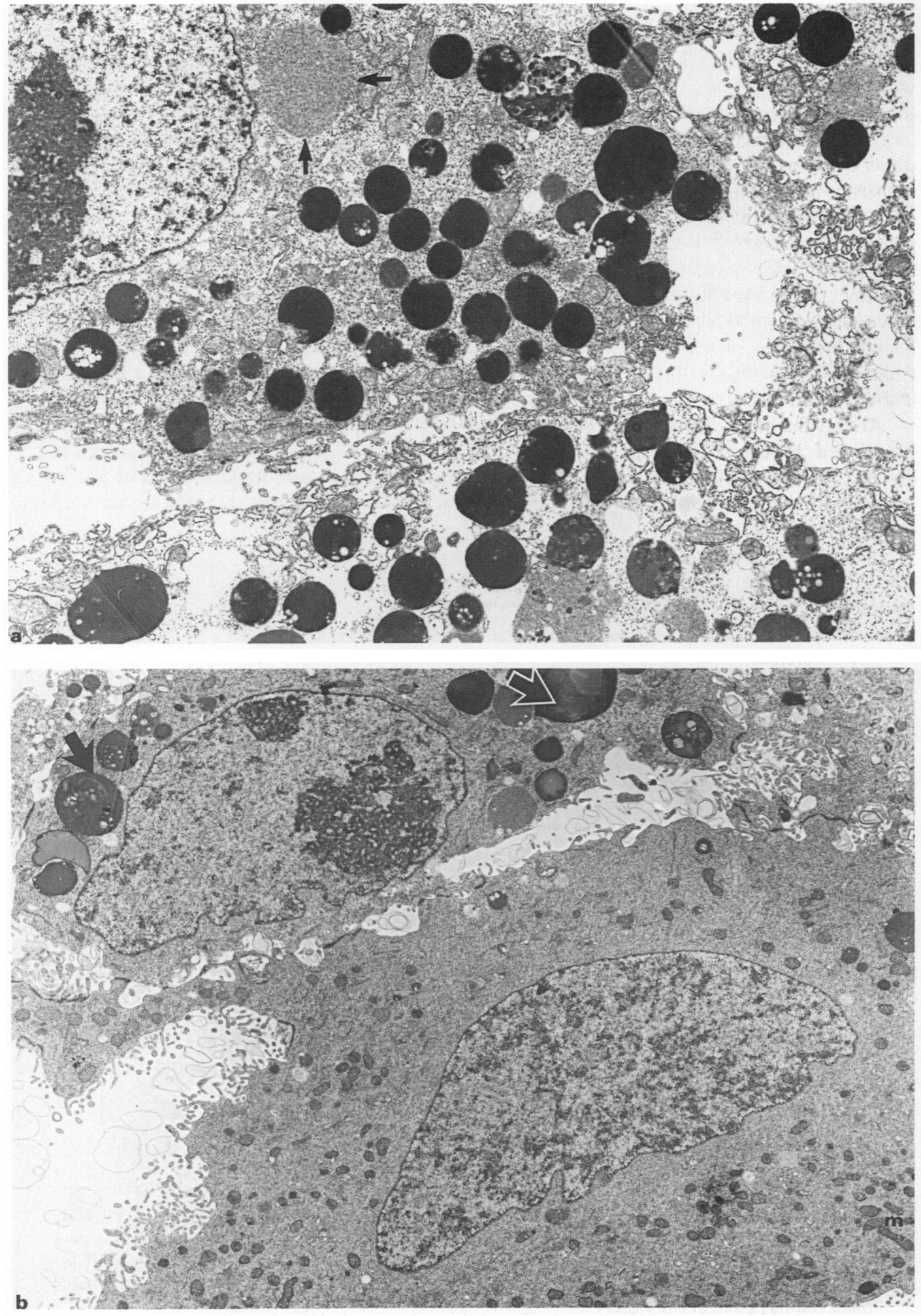
Failure to establish a proper chorioallantoic circulation between Days $7 \cdot 5$ and 8.5 of gestation (Theiler, 1972) would offer a simple explanation for death of $M$. caroli embryos in $M$. musculus. However, our studies at the light microscopic level suggest that the chorion had fused normally with the allantois. Similarly, the yolk sac placenta, an important nutritive and respiratory organ during early gestation, appeared to develop normally.

Trophoblast giant cells and ectoplacental cone cells in implantation sites from transferred $M$. caroli embryos were reduced in number compared to control embryos. Since ectoplacental cone cells eventually differentiate into spongiotrophoblast cells (Snell \& Stevens, 1966; Gardner et al., 1973; Rossant et al., 1983), paucity of these cells at Day 8.5 of gestation could adversely affect placental development and, subsequently, function. A deficiency of phagocytic trophoblast giant cells would limit the nutrients provided to the embryo (Robertson et al,, 1971; Poelmann \& Mentink, 1982), while lack of a cohesive network of trophoblast giant cells would readily allow cells and potentially harmful substances to cross Reichert's membrane and reach the embryo. This could include substances found in the uterus during normal pregnancy that are usually bound or modified by trophoblast cells. Alternatively, substances could escape from the embryonic cavity, altering embryo metabolism and disrupting proper materno-fetal interactions.

At Day 8.5 of gestation, numerous trophoblast cells, principally mural giant cells surrounding transferred $M$. caroli embryos, were degenerate as indicated by pycnosis, karyorrhexis and illdefined, vacuolated cytoplasm. This observation is significant because it precedes the earliest known participation of immune effector cells in $M$. caroli embryo failure (Croy et al., 1982; Clark et al., 1983). Tachi \& Tachi (1979) observed that trophoblast cells from rat embryos transferred to the $M$. musculus uterus degenerate and die shortly after penetration of the basement membrane of the uterine luminal epithelium. As in the present study, immune effector cells did not appear to be responsible for degeneration of xenogeneic rat trophoblast cells and similar mechanisms may be responsible for embryo death in both model systems.

The differences in the quantity and quality of cytoplasmic granules between trophoblast cells of $M$. musculus and $M$. caroli embryos recovered from $M$. musculus uterus were striking and are likely to be due to metabolic differences. The abundance of lipid inclusions in $M$. caroli trophoblast suggests low levels of metabolic activity consistent with an hypothesis of functional impairment of trophoblast. The substances contained within the granules of healthy $M$. caroli and $M$. musculus trophoblast cells are not known but most appear to be lysosomes or secretory bodies. The absence of histochemically detectable acid phosphatase activity in these trophoblast cells (data not shown) may indicate that lysosomal activity is not elevated in trophoblast as suggested by Welsh \& Enders (1987). The granules appear to be proteinaceous since they stain intensely with eosin but only moderately with periodic acid-Schiff. Since peptide hormones are known to be secreted by trophoblast throughout gestation (Robertson et al., 1982; Linzer \& Nathans, 1985; Linzer et al., 1985; Soares et al., 1985), many granules may contain hormones. The relationship between granule paucity in trophoblast from transferred $M$. caroli embryos and pregnancy failure is unclear. The contents of the granules may be essential for pregnancy, in which case failure to produce them would contribute directly to embryonic death. Alternatively, impairment of trophoblast development and function may result in reduced metabolic activity and reduction in the number of granules as a secondary problem.

Fig. 4. Electron micrographs of trophoblast cells. (a) M. musculus in M. musculus at Day $8 \cdot 5$ showing two trophoblast cells with granules of various sizes and appearance. Electron-dense granules are the most abundant. Arrow indicates phagocytosed erythrocyte. $\times 5100$. (b) $M$. caroli embryo from the same $M$. musculus recipient at Day 8.5. The upper trophoblast cell contains few inclusions, most of which are of intermediate electron density (arrow), while the lower cell has very few inclusions but many noticeable mitochondria. $\times 3400$. 

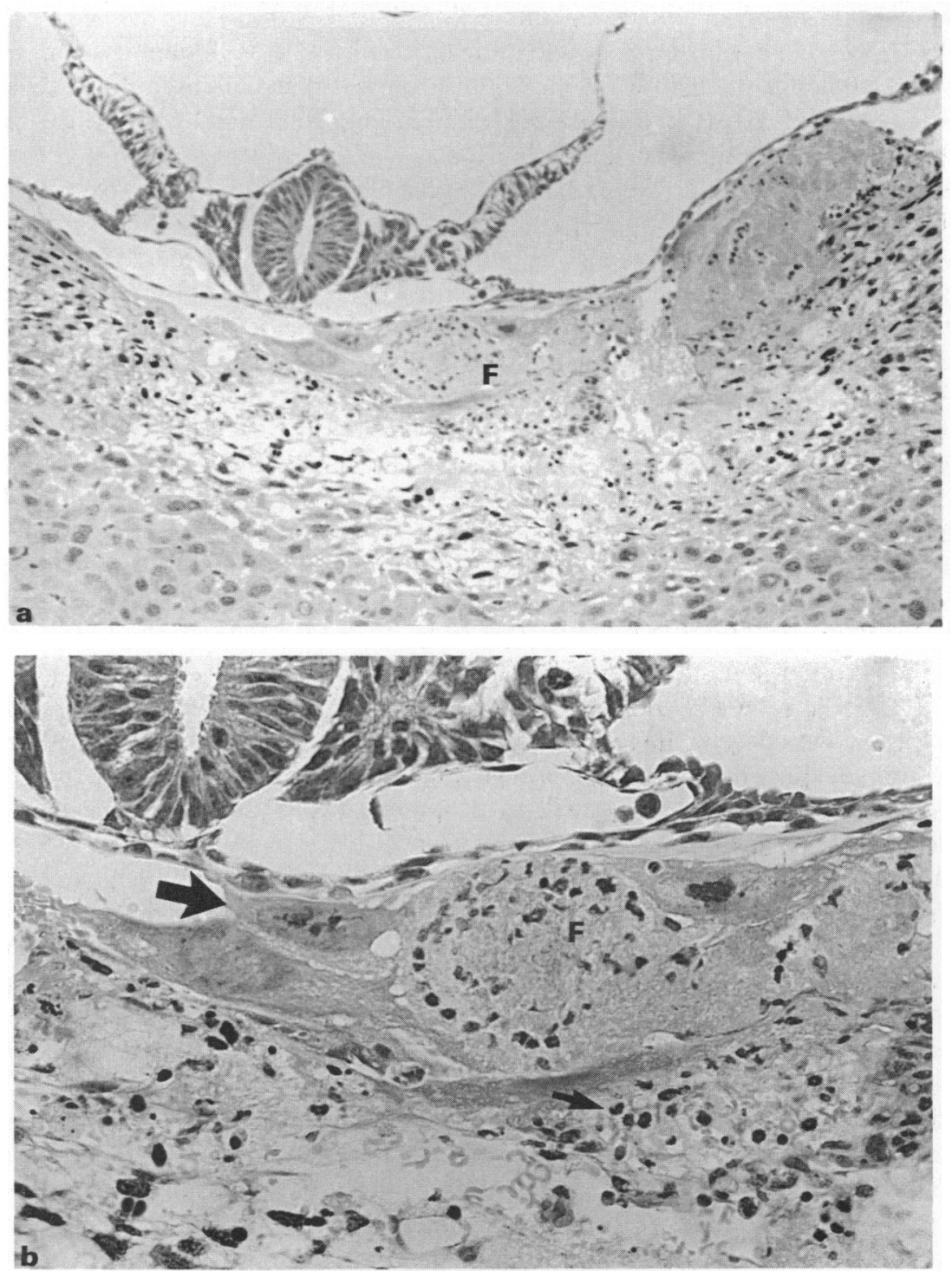

Fig. 5. Photomicrographs of $M$. caroli embryo in $M$. musculus uterus at Day 8.5 showing (a) exudative foci $(F)$ in the region of the posterior neural tube $(\times 128)$; and $(b)$ degenerating trophoblast cells (large arrow) adjacent to exudate $(F)$ and polymorphs (small arrow) $(\times 320)$.

Trophoblast giant cells are known to be resistant to immune cell-mediated lysis (Simmons \& Russell, 1962), even under conditions of enhanced immune effector activity (Zuckerman \& Head, 1985) or xenotransfer (Kirby, 1962; Hancock et al., 1968; Croy et al., 1984). Consequently, the observed degeneration of trophoblast giant cells around transferred $M$. caroli embryos is probably not mediated by immunological effector mechanisms. A role for lymphokines, particularly granulocyte-macrophage colony stimulating factor, has been postulated in trophoblast proliferation (Athanassakis et al., 1987) and it is possible that failure of $M$. caroli embryonic cells to stimulate $M$. musculus lymphokine production or to bind lymphokines of $M$. musculus origin remains as an immunological explanation for gradual degeneration of $M$. caroli trophoblast cells in xenogeneic pregnancy. 
However, the hypothesis that death of $M$. caroli embryos in $M$. musculus uteri is not immunologically mediated but initiated by trophoblast failure offers an explanation for lack of success in prolonging the survival of $M$. caroli embryos in immunodeficient compared to immunocompetent M. musculus recipients (Croy et al., 1985). Necrosis of trophoblast would recruit specific and non-specific immune effector cells and phagocytes to implantation sites and lack of trophoblast continuity would readily permit access of these cells to the embryo proper, before onset of gross degenerative changes.

Primary trophoblast failure and cell death also explain other observations made in this model system. Some $M$. caroli $<->M$. musculus aggregation chimaeras transferred to $M$. musculus uteri survive to term (Rossant et al., 1982). The trophoblast giant cells of the survivors are solely of $M$. musculus genotype by Day 9 of gestation (Rossant et al., 1983). Many chimaeras die during gestation and it can be suggested that the dying embryos are those which differentiate trophoblast cells wholly or largely of $M$. caroli genotype, while in the survivors gradual death and loss of $M$. caroli trophoblast cells from the mixed population results in a conceptus having trophoblast cells of the genotype of the embryo transfer recipient and survival. A relative deficiency of healthy $M$. caroli trophoblast cells could also account for the deficit in recruitment of decidua-associated, trophoblast-recruited, suppressor cells (Clark et al., 1983) at $M$. caroli implantation sites in M. musculus.

In many studies, lymphocytes have been deemed to play an important role in initiation of interspecies and intraspecies embryo death (Allen, 1982; Allen et al., 1986; Clark et al., 1986; Gronvik et al., 1986; Gendron \& Baines, 1988; Tartakovsky \& Gorelik, 1988). Failure to prolong $M$. caroli embryo survival in immunodeficient $M$. musculus recipients, including xenogeneically unresponsive recipients of scid/scid genotype (Croy et al., 1985; Crepeau \& Croy, 1988), and demonstration of trophoblastic failure early during gestation indicate that immunological differences between $M$. musculus and $M$. caroli are not responsible for initiation of pregnancy failure in this model system. Rather, immune effector cells appear to be recruited following degeneration of $M$. caroli trophoblast cells in the xenogeneic uterus and participate in embryonic resorption as a secondary event. Further detailed studies of other reported models of immunologically-mediated pregnancy failure, including histological, time course studies, may also show that presently unsuspected events underlie some or all of these embryonic deaths.

Support for these investigations was provided by the Natural Sciences and Engineering Research Council of Canada and the Ontario Ministry of Agriculture and Food. M.C. is the recipient of an Ontario Graduate Student Trainee award. We thank Christine Chapeau and Tim Bast for technical assistance.

\section{References}

Adamson, E.D. (1987) Review article: expression of proto-oncogenes in the placenta. Placenta 8, 449-466.

Allen, W.R. (1982) Immunological aspects of the equine endometrial cup reaction and the effect of xenogeneic extraspecies pregnancy in horses and donkeys. $J$. Reprod. Fert., Suppl. 31, 57-94.

Allen, W.R., Kydd, J.H. \& Antczak, D.F. (1986) Successful applications of immunotherapies to a model of pregnancy failure in equids. In Reproductive Immunology 1986, pp. 253-260. Eds D. A. Clark \& B. A. Croy. Elsevier Science Publishers, Amsterdam.

Anderson, G.B. (1988) Interspecific pregnancy: barriers and prospects. Biol. Reprod. 38, 1-15.

Athanassakis, I., Bleackley, R.C., Paetkau, V., Guilbert, L., Barr, P.J. \& Wegmann, T.G. (1987) The immunostimulatory effect of $T$ cells and $T$ cell lymphokines on murine fetally derived placental cells. J. Immunol. 138, 37-44.

Bennett, H.S., Wyrick, A.D., Lee, S.W. \& McNeil, J.H. (1976) Science and art in preparing tissues embedded in plastic for light microscopy, with special reference to glycol methacrylate, glass knives and simple stains. Stain Technol. 51, 71-97.

Chaouat, G., Kolb, J.P. \& Wegmann, T.G. (1983) The murine placenta as an immunological barrier between the mother and the fetus. Immunological Rev. 75, 31-60.

Clark, D.A., Slapsys, R.M., Croy, B.A. \& Rossant, J. (1983) Suppressor cell activity in uterine decidua correlates with success or failure of murine pregnancies. J. Immunol. 131, 540-542.

Clark, D.A., Chaput, A. \& Tutton, D. (1986) Active 
suppression of host-versus-graft reaction in pregnant mice. VII. Spontaneous abortion of allogeneic DBA/ $2 \times \mathrm{CBA} / \mathrm{J}$ fetuses in the uterus of CBA/J mice correlates with deficient non-T suppressor cell activity. $J$. Immunol. 136, 1668-1675.

Crepeau, M.A. \& Croy, B.A. (1988) Specific cellular immunity cannot account for failure of Mus caroli embryos transferred to Mus musculus of genotype scid/scid. Transplantation 45, 1104-1110.

Crepeau, M.A., Yamashiro, S. \& Croy, B.A. (1988) Anatomical and immunological aspects of fetal death in the Mus musculus/Mus caroli model of pregnancy failure. In Early Pregnancy Loss: Mechanisms and Treatment, pp. 69-83. Eds R. W. Beard \& F. Sharp. The Royal College of Obstetricians and Gynaecologists, London.

Croy, B.A., Rossant, J. \& Clark, D.A. (1982) Histological and immunological studies of post implantation death of Mus caroli embryos in the Mus musculus uterus. J. Reprod. Immunol. 4, 277-293.

Croy, B.A., Rossant, J. \& Clark, D.A. (1984) Recruitment of cytotoxic cells by ectopic grafts of xenogeneic, but not allogeneic, trophoblast. Transplantation, 37, 84-90.

Croy, B.A., Rossant, J. \& Clark, D.A. (1985) Effects of alterations in the immunocompetent status of $\mathrm{Mus}$ musculus females on the survival of transferred Mus caroli embryos. J. Reprod. Fert. 74, 479-489.

Fehilly, C.F. \& Willadsen, S.M. (1986) Embryo manipulation of farm animals. Oxford Rev. Reprod. Biol. 8, $317-413$

Frels, W.I., Rossant, J. \& Chapman, V.M. (1980) Intrinsic and extrinsic factors affecting the development of hybrids between Mus musculus and Mus caroli. J. Reprod. Fert. 59, 387-392.

Gardner, R.L., Papaioannou, V.E. \& Barton, S.C. (1973) Origin of the ectoplacental cone and secondary giant cells in mouse blastocysts reconstituted from isolated trophoblast and inner cell mass. J. Embryol. exp. Morph. 30, 561-572.

Gendron, R.L. \& Baines, M.G. (1988) Infiltrating decidual Natural Killer cells are associated with spontaneous abortion. Cell. Immunol. 113, 261-267.

Gronvik, K.O., Hoskin, D.W. \& Murgita, R.A. (1986) Monoclonal antibodies against murine neonatal and pregnancy-associated natural suppressor cells induce resorption of the fet us. Scand.J. Immunol. 25, 533-540.

Hancock, J.L., McGovern, P.T. \& Stamp, J.T. (1968) Failure of gestation of goat $\times$ sheep hybrids in goats and sheep. J. Reprod. Fert., Suppl. 3, 29-36.

Hunziker, R.D. \& Wegmann, T.G. (1987) Placental immunoregulation. CRC Reviews of Immunology 6, $245-285$.

Karnovsky, M.J. (1965) Formaldehyde-glutaraldehyde fixative of high osmolarity for use in electron microscopy. J. Cell Biol. 27, 137.

Kirby, D.R.S. (1962) Reciprocal transfer of blastocysts between rats and mice. Nature, (Lond.) 194, 785-786.

Linzer, D.I.H. \& Nathans, D. (1985) A new member of the prolactin-growth hormone gene family expressed in mouse placenta. $E M B O J .4,1419-1423$.

Linzer, D.I.H., Lee, S.J., Talamantes, F. \& Nathans, D. (1985) Identification of proliferin mRNA and protein in mouse placenta. Proc. nat. Acad. Sci. USA 82, 43564359.

Poelman, R.E. \& Mentink, M.M. (1982) Parietal yolk sac in early gestation mouse embryos: structure and function. In Structural and Functional Organization of the Placenta, Vol. 22, pp. 123-127. Eds P. Kaufman \& B. F. King. Bibliotheca Anatomica, S. Karger, Basel.

Polzin, V.J., Anderson, D.L., Anderson, G.B., BonDurant, R.H., Butler, J.E., Pashen, R.L., Penedo, M.C.F. \& Rowe, J.D. (1987) Production of sheep-goat chimeras by inner cell mass transplantation. J. Anim. Sci. 65, 325-330.

Robertson, M.C., Gillepsie, B. \& Friesen, H.G. (1982) Characterization of the two forms of rat placental lactogen (rPL): rPL-I and rPL-II. Endocrinology 111, [862-1866.

Robertson, T.A., Archer, J.M., Papaioannou, J.M. \& Walters, M.N-I. (1971) Transport of horseradish peroxidase in the murine placenta. J. Pathol. 103, 141-147.

Rossant, J., Mauro, V.M. \& Croy, B.A. (1982) Importance of trophoblast genotype for survival of interspecific murine chimeras. J. Embryol. exp. Morph. 69, 141-149.

Rossant, J., Croy, B.A., Clark, D.A. \& Chapman, V.M. (1983) Interspecific hybrids and chimeras in mice. $J$. exp. Zool. 228, 223-233.

Sherman, M.I. (1983) Endocrinology of rodent trophoblast cells. In Biology of Trophoblast, pp. 401-467. Eds Y. W. Loke \& A. Whyte. Elsevier Science Publishers, Amsterdam

Simmons, R.L. \& Russell, P.S. (1962) The antigenicity of mouse trophoblast. Ann. N.Y. Acad. Sci. 99, 717-732.

Snell, G.D. \& Stevens, L.C. (1966) Early embryology. In Biology of the Laboratory Mouse, pp. 205-246. Ed. E. L. Green. McGraw-Hill, New York.

Soares, M.J., Julian, J.A. \& Glasser, S.R. (1985) Trophoblast giant cell release of placental lactogens: temporal and regional characteristics. Devl Biol. 107, $520-526$.

Surani, M.A.H., Barton, S.C. \& Norris, M.L. (1987) Experimental reconstruction of mouse eggs and embryos: an analysis of mammalian development. Biol. Reprod. 36, 1-16.

Tachi, M.A. \& Tachi, C. (1979) Ultrastructural studies on maternal-embryonic cell interactions during experimentally induced implantation of rat blastocysts to the endometrium of the mouse. Devl Biol. 68, $203-223$.

Tartakovsky, B. \& Gorelik, E. (1988) Immunization with a syngeneic regressor tumor causes resorption in allopregnant mice. J. Reprod. Immunol. 13, 113-122.

Theiler, K. (1972) The House Mouse. Springer-Verlag, New York.

Welsh, A.D. \& Enders, A.E. (1987) Trophoblast-decidual cell interactions and establishment of maternal blood circulation in the parietal yolk sac of the rat. Anat. Rec. 217, 203-219.

Zuckermann, F.A. \& Head, J.R. (1985) Susceptibility of mouse trophoblast to antibody and complement mediated damage. Transplant. Proceed. 17, 925-927.

Received 30 August 1988 